\title{
An Adaptive Genetic Wavelet Neural Network and Its Application in Risk Assessment for Controlled Flight into Terrain
}

\author{
Xu-Sheng Gan ${ }^{1,2, a}$, Deng-Kai Yao ${ }^{1,2, b}$, Ya-Rong Wu ${ }^{1,2, c}$, Zhe Dai ${ }^{1,2, d}$ \\ ${ }^{1}$ Air Traffic Control and Navigation College, Air Force Engineering University, Xi'an, Shaanxi, \\ 710051 , China \\ ${ }^{2}$ National Key Laboratory of Air Traffic Collision Prevention, Xi'an, Shaanxi, 710051, China \\ a ganxusheng123@163.com, ${ }^{b}$ dk_yao1968@163.com, ${ }^{c}$ chuhs@126.com, ${ }^{d}$ z_dai1984@126.com
}

Keywords: Controlled flight into terrain; Risk Assessment; Genetic algorithm; Wavelet neural network

\begin{abstract}
In order to improve the level of safety management and decision-making of Controlled Flight Into Terrain (CFIT), a CFIT risk assessment model based on an adaptive genetic algorithm and wavelet neural network technology is propose. In the model, wavelet neural network can dynamically adjust the parameters of the network by learning the samples, the adjustment of these parameters can effectively reflect the change of each influence factor, and an adaptive genetic algorithm is introduced to solve the selection problem of initial network parameters. The practical example shows that the proposed model has a better assessment performance for CFIT risk.
\end{abstract}

\section{Introduction}

Controlled Flight Into Terrain (CFIT) is that, when the aircraft is fully in the airworthy condition, the pilot unintentionally manipulates the aircraft into the terrain, obstacles or water face. This kind of accident usually occur in the approach and landing phase, for large jet aircraft, the phase accounts for about $16 \%$ of the entire flight phase [1].

CFIT is one of the main types of flight accident in military and civil aviation at present, effecting the flight safety level directly. In order to prevent CFIT better, taking the system safety concept as guidance, learning from the advanced experiences in other fields, combining the theory and practice of CFIT safety, we introduce an adaptive genetic wavelet neural network to discuss the feasibility of its application in CFIT risk assessment.

At present, wavelet neural network is usually used to solve various problems, and attracts the wide attention. In the application of wavelet neural network, an important issue is to determine the initial value of dilation factor, translation factor and connection weight of the network. The improper selection of initial parameters not only affect the convergence speed of the network but also have a great influence on the performance of the network, directly related to the accuracy of the prediction results. In order to solve this problem, an adaptive genetic algorithm is used to optimize the initial parameters of wavelet neural network. An actual example of CFIT risk assessment validates its feasibility.

\section{Basic idea of adaptive genetic wavelet neural network}

The main modeling mechanism of adaptive genetic wavelet neural network is that, first use the global search ability of genetic algorithm to carry out the pre-learning on global rough accuracy for the dilation factor, translation factor and connection weight of wavelet neural network, locating the region of optimal solution, which makes the population that is compose of dilation factor, translation factor and connection weight aggregated in a few locations of parameter solution space, and then apply the local optimal capability of BP algorithm to perform the gradient careful search in these small solution spaces, obtaining the optimal solution. Therefore, the learning of adaptive genetic wavelet neural network is divided into two parts: 1 . Optimize the initial parameters of the network using adaptive genetic algorithm; 2. Train the wavelet neural network by BP algorithm after 
initialization of model.

\section{Adaptive genetic algorithm}

In order to ensure the uniformity and diversity that the solutions in the population distribute in space for the avoidance of premature convergence or non-convergence, we can adopt the adaptive genetic algorithm [2]. In the algorithm, the mutation probability $P_{m}$ can automatically be adjusted according to the formula (1). When genetic algorithm tends to be premature, the mutation probability should be increased to obtain the diversity of population. We can judge whether the algorithm tends to be premature according to the formula (1). The larger the different rate of the population, the better the diversity of the population; the smaller the different rate of the population, less the diversity of the population.

$$
d_{l}=\frac{\sum_{i=0}^{N} \sum_{\substack{k=0 \\ k \neq i}}^{N} f\left(a_{i}, b_{k}\right)}{(N-1)^{2} \cdot l} \times 100 \%
$$

where $N$ is the total number of the individuals in the population, $l$ is the length of individual gene chain, $f\left(a_{i}, b_{k}\right)$ is the different degree between the individual $a, b$, the formula of different degree between any 2 individual $a, b$ in the population is as follows

$$
f(a, b)=\sum_{j=0}^{l-1} a_{j} \oplus b_{j}
$$

where $a_{j}$ denotes $j$-th gene on chain gene of the individual $a ; b_{j}$ denotes $j$-th gene on chain gene of the individual $b$.

The algorithm is described as follows:

1. Initialization. The total number of individual in the population is $N$, the crossover probability $P_{c}=P_{c 0}$, the mutation probability $P_{m}=P_{m 0}$;

2. carry out the statistic for the different rate of individuals in the population, and calculate the fitness of each individual;

3. If the individuals in the population are better than that in gene warehouse, then these individuals preserve in gene warehouse; if there is no better individuals found, then release the excellent individuals in gene warehouse into the population;

4. Judge the different rate of the population

$$
d_{l}=\left\{\begin{array}{cc}
P_{m}=P_{m}+\Delta P_{m} & d_{l} \leq \sigma \\
P_{m}=P_{m 0} & d_{l}>\sigma
\end{array}\right.
$$

5. Perform the genetic operation such as selection, crossover and mutation;

6. The algorithm is terminated until satisfies the conditions such as maximal evolution times or network error.

\section{Wavelet neural network}

Wavelet neural network is recently a novel neural network developed by wavelet theory. It is based on wavelet theory with better integration of time-frequency localization characteristics of wavelet transform and self-learning function of neural network [3].

According to the definition of wavelet transform and discrete wavelet adverse transform, any function $f \in L^{2}(R)$ can be written as

$$
f=\sum_{m, n}^{\infty}\left\langle f, \tilde{\psi}_{m, n}(t)\right\rangle \psi_{m, n}(t)
$$

where $\tilde{\psi}_{m, n}(t)$ is the dual function of $\tilde{\psi}_{m, n}(t)$, and satisfies $\left\langle\psi_{m, n}, \tilde{\psi}_{j, k}\right\rangle=\delta_{m, i} \cdot \delta_{n, j}$. 
In the pratical calculation, the finite sum of polynomial expression in the formula (4) can effectively approximate the function $f \in L^{2}(R)$, i.e.

$$
\hat{f}=\sum_{m, n}^{N} w_{m, n} \psi_{m, n}(t)
$$

For the situation of Multi-Input and Multi-Output (MIMO), the structure of wavelet neural network is shown in Fig. 1. The output of $i$-th node in hidden layer is

$$
s_{j}=\sum_{i=1}^{I} w_{j i} x_{i}, \quad(j=1,2, \cdots, J)
$$

Then the $k$-th output of wavelet neural network is

$$
\hat{y}_{k}=\sum_{j=1}^{J} w_{k j} \psi\left(\frac{\sum_{i=1}^{I} w_{j i} x_{i}-b_{j}}{a_{j}}\right), \quad(k=1,2, \cdots, K)
$$

where $\psi(\cdot)$ is the wavelet function, $I$ is the number of nodes in input layer, $J$ is the number of nodes in hidden layer, $K$ is the number of nodes in output layer, $w_{j i}$ is the connection weight between $j$-th node of hidden layer and $i$-th node of input layer, $w_{k j}$ is the connection weight between $k$-th node of output layer and $j$-th node of hidden layer. In learning, in addition to the connection weight, the dilation factor $a_{j}$ and translation factor $b_{j}$ also need to be modified to accurately approximate the function.

\section{Realization of wavelet neural network based on adaptive genetic algorithm}

Steps of wavelet neural network algorithm based on adaptive genetic algorithm are described as follows:

1. Randomly generate a population that contains group of Gray floating bit string. Each bit string denotes a set of dilation factor, translation factor and connection weight in wavelet neural network;

2. Gray floating bit string is decoded as dilation factor, translation factor and connection weight, and operate the wavelet neural network to evaluate the network performance. The scale index of network performance is the error square sum between the network output value and the expected output value. The smaller the error square sum, the better the network performance;

3. Carry out the genetic operation, in order to generate next generation population and form next generation wavelet neural network;

4. Repeat the step 2 and 3 until meets the network error and maximal evolution times required, and decode the individual in final population, obtaining the dilation factor, translation factor and connection weight after the optimization of adaptive genetic algorithm;

5. The dilation factor, translation factor and connection weight optimized is used as the initial parameters of BP training algorithm of wavelet neural network. The steps of BP algorithm is same as that introduced in other literatures

\section{Practical example}

In the process of aviation system operation, there are many influence factors leading to CFIT risk [4][5]. According to SHELL theory and REASON model, we first establish the CFIT risk assessment index system as shown in Fig. 2, and then some experts invited conduct the assessment to 11 influence factors in terms of the assessment criteria of CFIT risk level in Table 1, finally we obtain 12 times assessment information (i.e. 12 samples) from the experts in Table 2, 'Score' is the CFIT risk assessment results. 10 samples are be selected as training sample and 2 samples as test sample. For 10 training samples, use wavelet neural network based on adaptive genetic algorithm to build the CFIT risk assessment assessment model, the training iteration times can be taken as 200. After the input of 
11-th and 12-th samples are imported in the model established, we can get the prediction output of 11 -th and 12-th samples, i.e. 0.75 and 0.92 , The result is consistent with the actual situation.

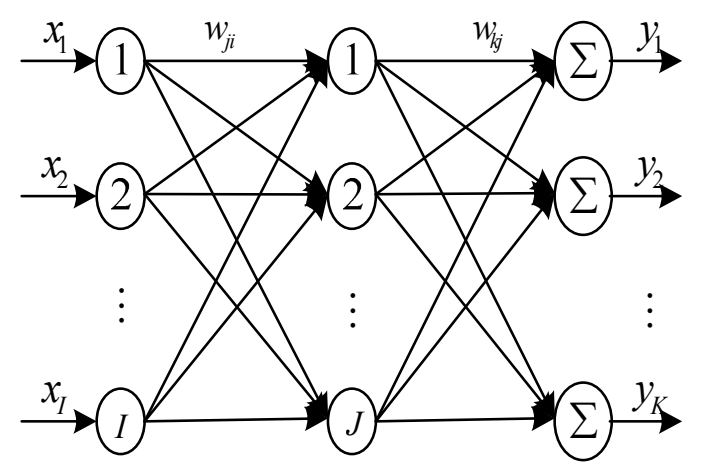

Fig. 1 wavelet neural network structure

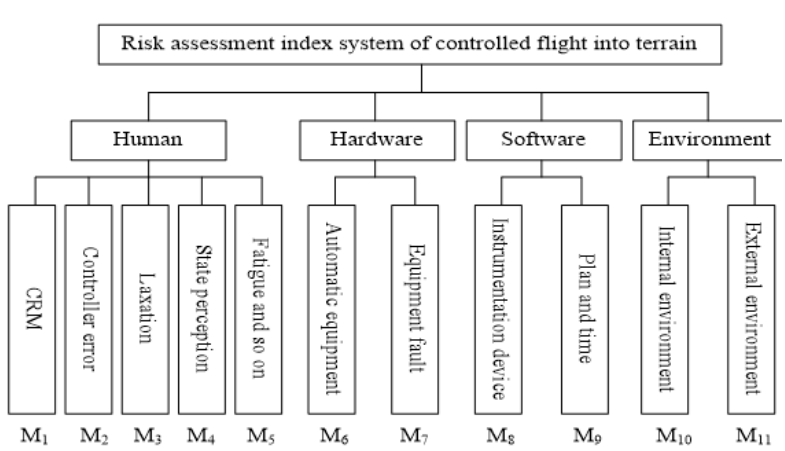

Fig. 2 CFIT risk assessment index system

Table 1 Assessment criteria of CFIT risk level

\begin{tabular}{cccccc}
\hline Score & $<0.6$ & $0.6 \sim 0.7$ & $0.7 \sim 0.8$ & $0.8 \sim 0.9$ & $>0.9$ \\
\hline Level & Unsafe & Unsafer & General & Safer & Safe \\
\hline
\end{tabular}

Table 2 CFIT risk assessment information of the experts

\begin{tabular}{c|cccccccccccc}
\hline Index & 1 & 2 & 3 & 4 & 5 & 6 & 7 & 8 & 9 & 10 & 11 & 12 \\
\hline$M_{1}$ & 0.67 & 0.54 & 0.69 & 0.73 & 0.75 & 0.85 & 0.69 & 0.69 & 0.86 & 0.86 & 0.81 & 0.82 \\
$M_{2}$ & 0.68 & 0.82 & 0.89 & 0.94 & 0.78 & 0.85 & 0.70 & 0.90 & 0.90 & 0.94 & 0.95 & 0.94 \\
$M_{3}$ & 0.62 & 0.71 & 0.69 & 0.79 & 0.78 & 0.80 & 0.59 & 0.83 & 0.93 & 0.88 & 0.91 & 0.92 \\
$M_{4}$ & 0.66 & 0.68 & 0.78 & 0.74 & 0.74 & 0.80 & 0.64 & 0.78 & 0.90 & 0.87 & 0.88 & 0.89 \\
$M_{5}$ & 0.74 & 0.72 & 0.88 & 0.80 & 0.73 & 0.77 & 0.70 & 0.75 & 0.85 & 0.87 & 0.90 & 0.91 \\
$M_{6}$ & 0.72 & 0.79 & 0.80 & 0.79 & 0.84 & 0.88 & 0.68 & 0.92 & 0.96 & 0.94 & 0.94 & 0.75 \\
$M_{7}$ & 0.69 & 0.82 & 0.89 & 0.95 & 0.78 & 0.82 & 0.72 & 0.92 & 0.91 & 0.94 & 0.95 & 0.96 \\
$M_{8}$ & 0.88 & 0.82 & 0.87 & 0.72 & 0.84 & 0.85 & 0.85 & 0.80 & 0.96 & 0.89 & 0.92 & 0.94 \\
$M_{9}$ & 0.63 & 0.69 & 0.79 & 0.74 & 0.73 & 0.82 & 0.60 & 0.78 & 0.92 & 0.87 & 0.85 & 0.86 \\
$M_{10}$ & 0.58 & 0.72 & 0.86 & 0.61 & 0.77 & 0.87 & 0.52 & 0.80 & 0.94 & 0.90 & 0.91 & 0.95 \\
$M_{11}$ & 0.65 & 0.56 & 0.69 & 0.73 & 0.75 & 0.84 & 0.66 & 0.68 & 0.86 & 0.86 & 0.81 & 0.80 \\
\hline Score & 0.75 & 0.73 & 0.85 & 0.79 & 0.77 & 0.89 & 0.75 & 0.77 & 0.97 & 0.88 & 0.77 & 0.89 \\
\hline
\end{tabular}

\section{Conclusions}

For the problem of CFIT management, an adaptive genetic wavelet neural network is proposed to establish CFIT risk assessment model. In the model, an adaptive genetic algorithm is used to optimize initial parameters of wavelet neural network for the avoidance uncertainty. The example shows that the proposed CFIT risk assessment model gives a satisfactory result.

\section{References}

[1] D. A. Smith, L. Wang. Controlled flight into terrain.Beijing: China Civil Aviation Publishing House, (2003),52-229

[2] Q. C.Wen, H.Peng, Q. Li. Application of adaptive genetic BP neural network model on statistical modeling. Microcomputer Information, 22(10-3), (2006), 130-132

[3] Q. H. Zhang, A. Benveniste. Wavelet networks. IEEE Trans. Neural Networks, 3, (1992), 889-898

[4] M. Janic. An assessment of risk and safety in civil aviation. Journal of Air Transport Management, 6(1), (2000), 43-50

[5] R. S. Sun, H. H. Liu. Assessment theory and practice for airline safety. China Safety Science Journal, 9(3), (1999), 69-73 